\title{
CME: Renal medicine (130893): self-assessment questionnaire
}

\author{
Authors: Edited by Michael Almond and Tahseen A Chowdhury
}

DOI: $10.7861 /$ clinmed.SAQ.20.2

\section{SAQs and answers are ONLINE for RCP fellows} and collegiate members

\section{Format}

Candidates are asked to choose the best answer from the five possible answers. This best of five format is used in many medical examinations; however, the questions are not intended to be representative of those used in the MRCP(UK) Part 1 or Part 2 Written Examinations.

\section{The answering process}

1 Go to www.rcplondon.ac.uk/SAQ

2 Log on using your usual RCP username and password

3 Select the relevant CME question paper

4 Answer all 10 questions by selecting the best answer from the options provided

5 Once you have answered all the questions, click on Submit

\section{Registering your external CPD credits}

Carrying out this activity allows you to claim two external CPD credits. These will be automatically transferred to your CPD diary, where you can review the activity and claim your points.

1. Which of the following was found to be true regarding the different modalities of renal replacement therapy?

(a) Haemodialysis has a survival advantage in older patients compared to peritoneal dialysis patients.

(b) Peritoneal dialysis has a survival advantage in older patients compared to haemodialysis patients.

(c) Peritoneal dialysis patients were found to have worse treatment satisfaction scores compared to haemodialysis.

(d) The rate of cognitive decline was found to be faster in peritoneal dialysis compared with haemodialysis.

(e) There was no survival advantage identified between haemodialysis and peritoneal dialysis in older patients.

2. Which of the following is true regarding frail patients receiving renal replacement therapy?

(a) Frailty is restricted to older patients on dialysis.

(b) Targeting blood pressure of $<140 / 90 \mathrm{mmHg}$ has been shown to improve outcomes. (c) The prevalence of depression is similar to that of the general public.

(d) The symptom burden experienced is similar to that of patients with malignancy.

(e) There is no increased risk of fracture after a fall compared to the general public.

3. A 72-year-old man with heart failure with reduced ejection fraction was admitted with 2 weeks of increased orthopnoea, dyspnoea on exertion and ankle swelling. He was taking enalapril $5 \mathrm{mg}$ twice daily (bd), bisoprolol $2.5 \mathrm{mg}$ once daily (od), spironolactone $50 \mathrm{mg}$ od and furosemide $40 \mathrm{mg}$ bd. Blood pressure was $122 / 88 \mathrm{mmHg}$ and heart rate was 66 beats per minute. His pre-admission baseline creatinine of $100 \mu \mathrm{mol} / \mathrm{L}$ was increased to $144 \mu \mathrm{mol} / \mathrm{L}$. His potassium was $4.8 \mathrm{mmol} / \mathrm{L}$.

As well as changing oral furosemide to at least $80 \mathrm{mg}$ bd intravenous, which is the correct change to his medication?
(a) Increase bisoprolol to $5 \mathrm{mg}$ once daily.
(b) No change.
(c) Reduce enalapril to $2.5 \mathrm{mg}$ twice daily.
(d) Reduce spironolactone to $12.5 \mathrm{mg}$ once daily.
(e) Stop enalapril.

4. A 25-year-old man with no past medical history presented to emergency department with severe malaise. He was found to be anaemic with haemoglobin of $69 \mathrm{~g} / \mathrm{L}$ (normal range 130-180 g/L). Blood film showed evidence of microangiopathic haemolysis and a thrombotic microangiopathy was suspected.
Which of the following would be more suggestive of a diagnosis of thrombotic thrombocytopenic purpura rather than complement-mediated atypical haemolytic uraemic syndrome?
(a) ADAMTS13 activity $>50 \%$.
(b) Creatinine of $90 \mu \mathrm{mol} / \mathrm{L}$ (normal range 50-120 $\mu \mathrm{mol} / \mathrm{L}$ ).
(c) Family history of thrombotic microangiopathy.
(d) Platelet count of $90 \times 10^{9} / \mathrm{L}$ (normal range $\left.150-400 \times 10^{9} / \mathrm{L}\right)$
(e) Shistocytes on blood film. 
5. A 27-year-old woman with a background of chronic kidney disease stage $\mathrm{G} 4, \mathrm{~A} 3$ due to complementmediated atypical haemolytic uraemic syndrome, maintained in remission using eculizumab, presented to the emergency department with 4 hours of headache, vomiting and fever.

\section{Which of the following is true regarding eculizumab?}

(a) Contraindicated after renal transplant.

(b) Increased risk of infection with encapsulated organisms.

(c) Inhibits alternative pathway to prevent paroxysmal nocturnal haemoglobinuria.

(d) Mechanism of action is C1 inhibition.

(e) Migraine is a major side effect.

6. Which of these factors is a recognised risk factor for cancer in a kidney transplant recipient?
(a) B cell depleting agents.
(b) Epstein-Barr virus.
(c) Herpes simplex virus.
(d) Obesity.
(e) Past alcohol history.

7. Which of the following recommendations is correct regarding screening for post-transplant malignancies?

(a) Two-yearly colonoscopy for those aged 60-74 years old to detect colorectal cancer.

(b) Three-yearly Papanicolaou smears in female recipients.

(c) Annual positron-emission tomography to screen for posttransplant lymphoproliferative disorder.

(d) Annual ultrasound of native kidneys to detect renal cell cancer.

(e) No routine dermatology review is required.

8. Which of these drugs is associated with an increased risk of malignancy in the post-transplant setting?

(a) Azathioprine.
(b) Cotrimoxazole. (c) Mammalian target of rapamycin (mTOR) inhibitor.

(d) Prednisolone.

(e) Valganciclovir.

9. A patient with chronic kidney disease stage 3 (estimated glomerular filtration rate $49 \mathrm{~mL} / \mathrm{min} / 1.73 \mathrm{~m}^{2}$ ) is admitted to hospital and is known to have heparin-induced thrombocytopenia.

\section{Which form of heparin is safe to use if parenteral anticoagulation is required?}
(a) Dalteparin.
(b) Enoxaparin
(c) Fondaparinux.
(d) Tinzaparin.
(e) Unfractionated heparin.

10. A haemodialysis patient has suffered two unprovoked pulmonary emboli in the past year. It was decided that he requires long-term oral anticoagulation.

Which oral anticoagulant is currently licensed in the UK for use in this patient?
(a) Apixaban.
(b) Dabigatran
(c) Edoxaban.
(d) Rivaroxaban.
(e) Warfarin.

CME Cardiovascular medicine SAQ
Answers to the CME SAQ published in Clinical
Medicine in January 2020
Q1
Q2

